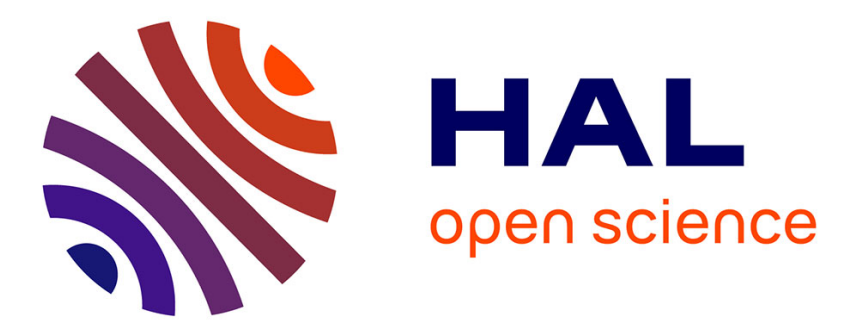

\title{
Highly-resolved separation of carrier and thermal wave contributions to photothermal signals from Cr-doped silicon using rate-window infrared radiometry
}

\author{
A. Mandelis, R. Bleiss
}

\section{- To cite this version:}

A. Mandelis, R. Bleiss. Highly-resolved separation of carrier and thermal wave contributions to photothermal signals from Cr-doped silicon using rate-window infrared radiometry. Journal de Physique IV Proceedings, 1994, 04 (C7), pp.C7-137-C7-140. 10.1051/jp4:1994733 • jpa-00253262

HAL Id: jpa-00253262

https://hal.science/jpa-00253262

Submitted on 1 Jan 1994

HAL is a multi-disciplinary open access archive for the deposit and dissemination of scientific research documents, whether they are published or not. The documents may come from teaching and research institutions in France or abroad, or from public or private research centers.
L'archive ouverte pluridisciplinaire HAL, est destinée au dépôt et à la diffusion de documents scientifiques de niveau recherche, publiés ou non, émanant des établissements d'enseignement et de recherche français ou étrangers, des laboratoires publics ou privés. 


\title{
Highly-resolved separation of carrier and thermal wave contributions to photothermal signals from $\mathrm{Cr}$-doped silicon using rate-window infrared radiometry
}

\author{
A. Mandelis and R. Bleiss $(1)$ \\ Photothermal and Optoelectronic Diagnostics Laboratory, Department of Mechanical Engineering, \\ University of Toronto, Toronto, Canada, M5S IA4
}

\begin{abstract}
We have shown that the new photothermal technique of lock-in rate-window infrared radiometry (RW-PTR) is capable of completely separating out photo-excited free-carrier-wave and thermalwave contributions to the photothermal signal from an n-type, Cr-doped $\mathrm{Si}$ wafer with a simple experimental procedure, and with superior temporal resolution in the determination of the electronic lifetime and thermal transport time constant.
\end{abstract}

\section{INTRODUCTION}

The measurement of photoexcited excess carrier lifetime is useful in characterizing the quality of semiconductor materials and modeling semiconductor devices. Besides the conventional photothermal techniques for lifetime measurements, photothermal radiometry [1] has proven to yield signals from semiconductors which are directly related to the density of photoexcited free carriers [2,3]. Recently we introduced the lock-in rate-window radiometric instrumentation and detection scheme, [4,5] which combines the extremely high dynamic reserve of frequency-domain narrow-band detection techniques with the simplicity of interpretation of time-resolved photothermal signals in terms of response delay times. It is well known that when used with semiconductors, the pulsed radiometric signal may contain both carrier recombination and thermal conduction components [2].

In Ref. 6 we showed that lock-in rate-window pulsed photothermal radiometry (RW-PTR) of deepimpurity-doped Si can yield excess photoexcited carrier bulk life-times with optimal signal-to-noise (S/N) ratio, from the simple, unambiguous interpretation of the maximum position of the rate-window signal. We also showed that measured lifetimes are mostly in good agreement with results obtained from the noncontact laser/microwave reflection detection method [7]. In this work we applied the lock-in RW-PTR to $\mathrm{Cr}$-doped $n$-type $\mathrm{Si}$, with preoxidized and etched high-quality surface, a sample which exhibits conduction transient behavior (Fig. 1). By choosing appropriate transient pulse durations(i.e., acoustooptically time gating a cw laser beam) for the first time we have been able to separate the two transient contributions completely and measure the respective delay-time constants with superior resolution to both frequency- and time-domain conventional methodologies. This ability of RW-PTR enormously simplifies and analytical complexity of conventional photothermal-based semiconductor diagnostic techniques, such as thermoreflectance [8] and frequency-domain PTR [2]. 

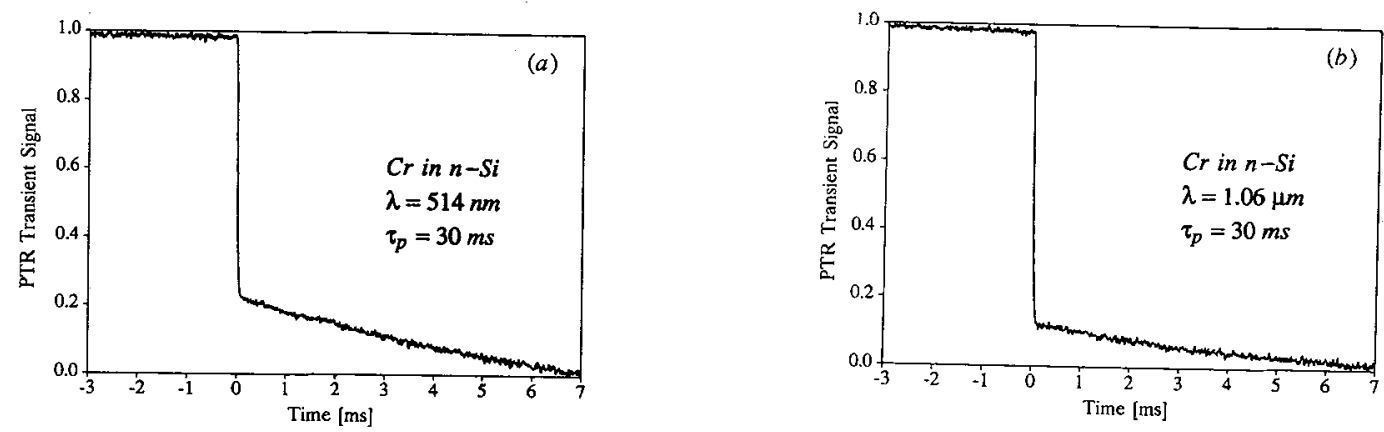

Fig. 1 PTR transient response of a Cr-doped $n$-type $S$ i wafer $\left(1.7 \times 10^{13} \mathrm{Cr}\right.$ atoms $\left./ \mathrm{cm}^{2} ; 5-13 \Omega \mathrm{cm}\right)$ to (a) an Ar-ion laser "pulse;" and (b) a Nd-YAG laser "pulse." Pulse duration $\tau_{p}=30 \mathrm{~ms}$.

\section{EXPERIMENTAL RESULTS AND DISCUSSIONS}

The experimental apparatus has been described previously $[5,6]$. Computer software was generated which enables the automatic acousto-optic scanning of the repetition period $T_{0}$ of the optical pulse, while keeping the pulse duration $\tau_{p}$ fixed at a predetermined value which, in turn, depended on the photothermal decay process being monitored. The two laser wavelengths chosen, $514 \mathrm{~nm}$ and $1.06 \mu \mathrm{m}$ ) were used to probe the surface $(\sim 0.5 \mu \mathrm{m})$ and the bulk $(\sim 1 \mathrm{~mm})$ of the Si wafer, respectively [9].

The infrared radiation from the sample was collected by two off-axis paraboloidal mirrors and detected using a liquid- $\mathrm{N}_{2}$-cooled mercury-cadmium telluride detector. Two experimental runs were performed at each wavelength, one with $\tau_{p}=30 \mu \mathrm{s}$ and a second run with $\tau_{p}=1 \mathrm{~ms}$. The temperature rises were estimated to be less than $1 \mathrm{~K}$ in both cases. Figure 2 shows the lock-in RW-PTR in-phase signal as a function of the laser time-gated square pulse repetition period $T_{0}$, resulting from a pulse of duration $\tau_{p}=$ $30 \mu \mathrm{s}$. In the lock-in rate-window method, one measures the in-phase (IP) signal resulting from the lock-in amplifier, which amounts to the fundamental Fourier component $b_{1}$ of the transient, repetitive PTR signal over the pulse duration $T_{o}$. When $T_{0}$ is scanned, an extremum occurs in $b_{1}$ corresponding to a particular $T_{o}$ which is roughly equal to the decay time characteristic of the photothermal process (in this case the carrier recombination lifetime or the thermal transit time across the semiconductor). The solid lines in Fig. 2 are the theoretical simulation using the expression for the fundamental Fourier component $b_{1}$ of the repetitive transient PTR signal $S_{\mathbb{R}}(t ; \tau)$ over the period $T_{0}$, using the fact that the fast decay in Fig. 1 is a purely exponential function [6].

The exponential PTR response assumes complete domination of the signal by the photoexcited free-carrier density kinetics, when the carrier surface recombination velocity is very low [6]. The excellent fits of the theory to the data, normalized to unity for convenience, prove the free-carrier interband recombination origin of the signal. No actual transient traces could be obtained in an oscilloscope for this short $\tau_{p}$, because the extremely small PTR signal could not be made to rise above the instrumental noise level, even after averaging several thousand transients [5]. It is important to note that the high sensitivity of the theoretical curve to $\tau$ in Figs. 2(a) and 2(b) renders the fit to the experimental data shown such that only true single-exponential recombination decays can yield the detailed agreement shown in Fig. 2, provided 
the PTR response is linear with the incident radiation intensity (which was the case with all our data). Any deviations from exponential decay manifest themselves as an inability to fit the entire data curve to a single exponential function.
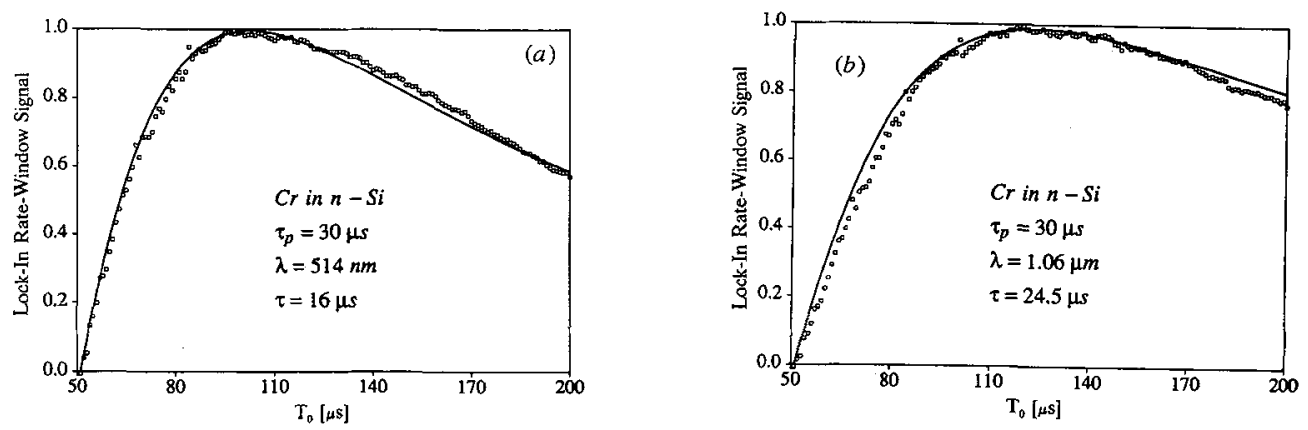

Fig. 2 Lock-in rate-window photothermal radiometric response to a repetitive square laser pulse of duration $\tau_{p}=30 \mu \mathrm{s}$. Squares: data; lines: theory, [6]. (a) $514 \mathrm{~nm}$ photon excitation of Cr-doped $n$-type Si wafer; (b) $1.06 \mu \mathrm{m}$ photon excitation.

The difference in lifetime $\tau$ between the results obtained with the Ar and Nd-YAG laser, Figs. 2(a) and 2(b), can be explained by the widely different optical penetration depth of the two excitation pulses. The $514 \mathrm{~nm}$ pulse probes a region very close to the surface, in which near-surface defects can provide an additional free-carier recombination channel, thus shortening the effective lifetime [10]. On the other hand, the deeply penetrating $1.06 \mu \mathrm{m}$ pulse is expected to give a better measurement of the true value of the bulk recombination lifetime. In this case near-surface recombination is much less significant in its contribution to the effective lifetime, and therefore the effective lifetime is longer and characteristic of bulk processes.

When the pulse duration was increased to $\tau_{p}=1 \mathrm{~ms}$, the lock-in RW-PTR response of the Cr-doped silicon wafer to the Ar laser excitation is shown in Fig. 3. This response, too, is normalized to unity for convenience. A very similar curve was obtained with Nd-YAG laser excitation. The maximum of the inphase signal at about $2 \mathrm{~ms}$ from the onset of the pulse is in good agreement with domination of the infrared radiometric signal by a thermal transient, which in the $500-\mu \mathrm{m}$-thick free-standing $\mathrm{Si}$ wafer requires a round-trip time [6]

$$
t_{\mathrm{st}} \approx L^{2} \pi / 4 \alpha_{s} \approx 1.96 \mathrm{~ms}
$$




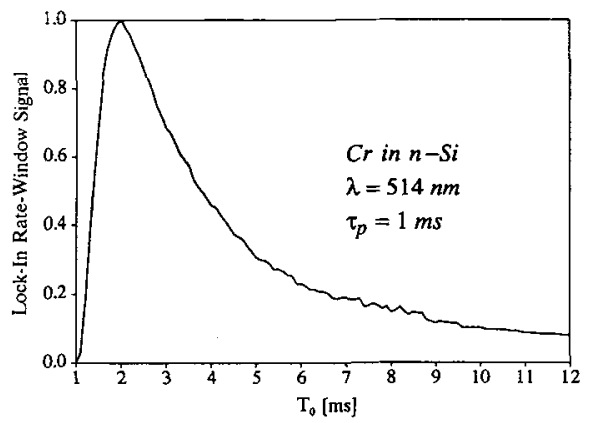

Fig. 3 Lock-in rate-window photothermal radiometric response of $\mathrm{Cr}$-doped Si wafer to a repetitive square Ar-laser pulse of duration $\tau_{p}=1 \mathrm{~ms}$.

The comparison between Figs. 2 and 3 shows the ability of RW photothermal detection to completely separate out electronic and thermal transport contributions to overlapping PTR signals from a Cr-doped Si wafer. This can be done with excellent temporal resolution, by a simple change in $\tau_{p}$. Similar results have been observed with $\mathrm{Fe}$-doped $\mathrm{Si}$ wafers as well. It is worth mentioning that the completely separate, highly-resolved results shown in Figs. 2 and 3 cannot be obtained either with frequency-scanned PTR detection, because of the time-multiplexed nature of this methodology, or with pulsed laser excitation, due to the fixed (i.e. non-continuously variable over long timescales) repetition rate and pulse duration of today's laser technologies.

\section{REFERENCES}

[1] Kanstad, S.O. and Nordal, P.-E., Powder Technol. 22, (1978) 55.

[2] Sheard, S.J. and Somekh, M.G., Infrared Phys. 28 (1988) 287.

[3] Cho, K. and Davis, C.C., IEEE J. Quant. Electron. QE-25, (1989) 1112.

[4] Mandelis, A. and Chen, Z.H., Rev. Sci. Instrum. 63 (1992) 2977.

[5] Chen, Z.H. and Mandelis, A., Phys. Rev. B46 (1992-II) 13526.

[6] Chen, Z.H., Bleiss, R., Mandelis, A., Buczkowski, A. and Shimura, F., J. Appl. Phys. 73 (1993) 5043.

[7] Shimura, F., Okui, T. and Kusama, T., Appl. Phys. 67 (1990) 7168.

[8] Rosencwaig, A. in Photoacoustic and Thermal-Wave Phenomena in Semiconductors, edited by A. Mandelis (Elsevier, New York, 1987), Chap. 5.

[9] Dash, W.C. and Neumann, R., Phys. Rev. 99 (1955) 1151.

[10] Ling, Z.G. and Ajmera, P.K., J. Appl. Phys. 69 (1991) 519. 